\title{
Application of A New Finite Volume Method to Burgers Equation
}

\author{
FANG Li ${ }^{1,}$, CUI Guo ${ }^{1, b}$ \\ ${ }^{1}$ Harbin Engineering University, P. R. China \\ a1396620409@qq.com, b9627204@qq.com
}

Keywords: Burgers equation, multiple integral finite volume method, Lagrange interpolation Abstract. In this paper, a new discrete scheme is constructed for nonlinear transient Burgers equation by using the multiple integral finite volume method combined with Lagrange interpolation. The scheme has third order accuracy in space. Of course, higher accuracy can be achieved by adding interpolation nodes. Numerical results show that current method is effective, and compared with others, it shows clear advantage in solution precision.

\section{Introduction}

The Burgers equation is a very important nonlinear equation. It is the most primitive model to describe the interaction between convection and diffusion. The equation combines the properties of first order wave equation and heat conduction equation. Therefore, further research on the Burgers equation is helpful to better solve other nonlinear partial differential equation models. The exact solution of the equation is feasible. But the calculation is large and the results are tedious, which is not conducive to further calculation (such as integral calculation). Therefore, it is significance to study the numerical method for Burgers equation.

All along, the numerical solution of Burgers equation has attracted lots of attention. Firstly, the Burgers equation was proposed by Bateman. Then Burger [1] used it to build a mathematical model. Until now, people have been exploring and studying the problem of solving Burgers equation. Due to the practical needs, numerical methods for Burgers equation are more popular. The numerical methods for Burgers equation include finite difference method, finite element method, boundary element method, finite volume method and so on. In recent years, the finite difference method of higher order compact difference scheme has attracted much attention, such as reference [3]. The finite volume method mentioned in reference [4] has also attracted the attention of scholars.

In this paper, a new finite volume method-multiple integral finite volume method for solving Burgers equation is presented.

In this paper, one-dimensional transient nonlinear Burgers equation is studied as follows:

$$
\begin{aligned}
& \frac{\partial u}{\partial t}+u \frac{\partial u}{\partial x}=\varepsilon \frac{\partial^{2} u}{\partial x^{2}}, 0 \leq x \leq 1,0<t \leq T . \\
& u(x, 0)=g(x), 0<x<1, \quad u(a, t)=w_{1}(t) \quad u(b, t)=w_{2}(t), \quad 0<t \leq T .
\end{aligned}
$$

in which, $g(x), w_{1}(t), w_{2}(t)$ are constant functions. $\varepsilon>0$ is the kinematic viscosity parameter. And the equation is consisted of a time-dependent term, a convection term and a diffusion term.

In this paper, the three integrals for Burgers equation are introduced firstly. The convection term, diffusion term, and time term are then treated separately. A scheme with three order accuracy in space is obtained. Finally, a numerical example is given to illustrate the feasibility of current method, and the precision of numerical results are compared with those obtained from other approaches.

\section{Construction of the discrete scheme}

Firstly, we divide the time region $0<t \leq T$ into $N$ subintervals. And time step is $\tau=t_{n+1}-t_{n}$, where 
$t_{n}=n \tau$ for $n=0,1,2 \mathrm{~L}, N$. Then we divide the space region $0 \leq x \leq 1$ into $J$ subintervals. In space, the step is $h=x_{i}-x_{i-1}$ where $x_{i}=i h$ for $i=1,2, \mathrm{~K} J$. So, we have $0=x_{0}<x_{1}<\mathrm{K}<x_{J}=1$. The quantity $u_{i}^{n}$ represents the numerical solution at $\left(x_{i}, t_{n}\right)$. We select $\left[x_{i}-\sigma h, x_{i}+\sigma h\right]$ as the control integral region. Here, $s_{1}$ and $s_{2}$ satisfy $x_{i}-\sigma h<s_{1}<s_{2}<x_{i}+\sigma h$ where $0<\sigma<1$.

We integrate the Burgers equations in the control volume,

$$
\int_{x_{i}}^{x_{i}+\sigma h} \int_{x_{i}-\sigma h}^{x_{i}} \int_{s_{1}}^{s_{2}}\left(\frac{\partial u}{\partial t}+u \frac{\partial u}{\partial x}-\varepsilon \frac{\partial^{2} u}{\partial x^{2}}\right) d x d s_{1} d s_{2}=0 .
$$

At any time level, Eq. (1) is tenable. In order to achieve second order accuracy in time, we discretize Eq.(3) on the $n+1 / 2$ layer.

$$
\int_{x_{i}}^{x_{i}+\sigma h} \int_{x_{i}-\sigma h}^{x_{i}} \int_{s_{1}}^{s_{2}}\left(\frac{\partial u^{n+\frac{1}{2}}}{\partial t}+u^{n+\frac{1}{2}} \frac{\partial u^{n+\frac{1}{2}}}{\partial x}-\varepsilon \frac{\partial^{2}\left(u^{n+\frac{1}{2}}\right)}{\partial x^{2}}\right) d x d s_{1} d s_{2}=0
$$

First, we discretize the convection term. We can have,

$$
\int_{x_{i}}^{x_{i}+\sigma h} \int_{x_{i}-\sigma h}^{x_{i}} \int_{s_{1}}^{s_{2}} u^{n+\frac{1}{2}} \frac{\partial\left(u^{n+\frac{1}{2}}\right)}{\partial x} d x d s_{1} d s_{2}=\frac{1}{2} \int_{x_{i}}^{x_{i}+\sigma h} \int_{x_{i}-\sigma h}^{x_{i}}\left[\left(u_{s_{2}}^{n+\frac{1}{2}}\right)^{2}-\left(u_{s_{1}}^{n+\frac{1}{2}}\right)^{2}\right] d s_{1} d s_{2} .
$$

By Taylor formula, we get the following,

$$
u^{n+1}=u^{n+\frac{1}{2}}+\frac{\tau}{2}\left(u^{n+\frac{1}{2}}\right)^{\prime}+\mathrm{O}\left(\tau^{2}\right), u^{n}=u^{n+\frac{1}{2}}-\frac{\tau}{2}\left(u^{n+\frac{1}{2}}\right)^{\prime}+\mathrm{O}\left(\tau^{2}\right), u^{n-1}=u^{n+\frac{1}{2}}-\frac{3 \tau}{2}\left(u^{n+\frac{1}{2}}\right)^{\prime}+\mathrm{O}\left(\tau^{2}\right) .
$$

and then ,

$$
u^{n+\frac{1}{2}}=\frac{u^{n+1}+u^{n}}{2}+\mathrm{O}\left(\tau^{2}\right)
$$

and,

$$
u^{n+\frac{1}{2}}=\frac{3 u^{n}-u^{n-1}}{2}+\mathrm{O}\left(\tau^{2}\right)
$$

therefore ,

$$
\left(u^{n+\frac{1}{2}}\right)^{2}=u^{n+\frac{1}{2}} \cdot u^{n+\frac{1}{2}}=\frac{u^{n+1}+u^{n}}{2} \cdot \frac{3 u^{n}-u^{n-1}}{2}+\mathrm{O}\left(\tau^{2}\right) .
$$

We can simplify the convection term into

$$
\begin{aligned}
& \int_{x_{i}}^{x_{i}+\sigma h} \int_{x_{i}-\sigma h}^{x_{i}} \int_{s_{1}}^{s_{2}} u^{n+\frac{1}{2}} \frac{\partial\left(u^{n+\frac{1}{2}}\right)}{\partial x} d x d s_{1} d s_{2} \\
= & \frac{\sigma h}{8}\left[\int_{x_{i}}^{x_{i}+\sigma h}\left(u_{s_{2}}^{n+1}+u_{s_{2}}^{n}\right)\left(3 u_{s_{2}}^{n}-u_{s_{2}}^{n-1}\right) d s_{2}-\int_{x_{i}-\sigma h}^{x_{i}}\left(u_{s_{1}}^{n+1}+u_{s_{1}}^{n}\right)\left(3 u_{s_{1}}^{n}-u_{s_{1}}^{n-1}\right) d s_{1}\right] .
\end{aligned}
$$

Since we cannot find the functions of $u_{s_{2}}^{n+1}, u_{s_{2}}^{n}, u_{s_{2}}^{n-1}, u_{s_{1}}^{n+1}, u_{s_{1}}^{n}$ and $u_{s_{1}}^{n-1}$. We utilize three-points Lagrange interpolation at $x_{i-1}, x_{i}$ and $x_{i+1}$ respectively, as shown follows

$$
u_{s_{2}}^{n+1}=\frac{\left(s_{2}-x_{i}\right)\left(s_{2}-x_{i+1}\right)}{\left(x_{i-1}-x_{i}\right)\left(x_{i-1}-x_{i+1}\right)} u_{i-1}^{n+1}+\frac{\left(s_{2}-x_{i-1}\right)\left(s_{2}-x_{i+1}\right)}{\left(x_{i}-x_{i-1}\right)\left(x_{i}-x_{i+1}\right)} u_{i}^{n+1}+\frac{\left(s_{2}-x_{i-1}\right)\left(s_{2}-x_{i}\right)}{\left(x_{i+1}-x_{i-1}\right)\left(x_{i+1}-x_{i}\right)} u_{i+1}^{n+1} \text {. }
$$

By substituting the interpolation polynomials of $u_{s_{2}}^{n+1}, u_{s_{2}}^{n}, u_{s_{2}}^{n-1}, u_{s_{1}}^{n+1}, u_{s_{1}}^{n}$ and $u_{s_{1}}^{n-1}$ into Eq.(10). We get,

$$
\int_{x_{i}}^{x_{i}+\sigma h} \int_{x_{i}-\sigma h}^{x_{i}} \int_{s_{1}}^{s_{2}} u^{n+\frac{1}{2}} \frac{\partial u^{n+\frac{1}{2}}}{\partial x} d x d s_{1} d s_{2}=A_{0} u_{i-1}^{n+1}+A_{1} u_{i}^{n+1}+A_{2} u_{i+1}^{n+1}+A_{3} .
$$

where $A_{0}=\frac{h^{2} \sigma^{5}}{32} u_{i-1}^{n-1}+\frac{h^{2} \sigma^{3}}{32}\left(2-\sigma^{2}\right) u_{i}^{n-1}-\frac{3 h^{2} \sigma^{5}}{32} u_{i-1}^{n}+\frac{h^{2} \sigma^{3}}{32}\left(3 \sigma^{2}-6\right) u_{i}^{n}$.

$$
A_{1}=\frac{h^{2} \sigma^{3}}{32}\left(2-\sigma^{2}\right) u_{i-1}^{n-1}+\frac{h^{2} \sigma^{3}}{32}\left(\sigma^{2}-2\right) u_{i+1}^{n-1}+\frac{h^{2} \sigma^{3}}{32}\left(3 \sigma^{2}-6\right) u_{i-1}^{n}+\frac{h^{2} \sigma^{3}}{32}\left(6-3 \sigma^{2}\right) u_{i+1}^{n} \text {. }
$$




$$
\begin{aligned}
A_{2} & =\frac{h^{2} \sigma^{3}}{32}\left(\sigma^{2}-2\right) u_{i}^{n-1}-\frac{h^{2} \sigma^{5}}{32} u_{i+1}^{n-1}+\frac{h^{2} \sigma^{3}}{32}\left(6-3 \sigma^{2}\right) u_{i}^{n}+\frac{3 h^{2} \sigma^{5}}{32} u_{i+1}^{n} . \\
A_{3} & =\frac{h^{2} \sigma^{5}}{32} u_{i-1}^{n-1} u_{i-1}^{n}+\frac{h^{2} \sigma^{3}}{32}\left(2-\sigma^{2}\right)\left(u_{i-1}^{n-1} u_{i}^{n}+u_{i}^{n-1} u_{i-1}^{n}\right)-\frac{3 h^{2} \sigma^{5}}{32}\left(u_{i-1}^{n}\right)^{2}+2 \frac{h^{2} \sigma^{3}}{32}\left(3 \sigma^{2}-6\right) u_{i-1}^{n} u_{i}^{n} \\
& +2 f_{1} u_{i}^{n} u_{i+1}^{n}+\frac{h^{2} \sigma^{3}}{32}\left(\sigma^{2}-2\right)\left(u_{i}^{n-1} u_{i+1}^{n}+u_{i+1}^{n-1} u_{i}^{n}\right)-\frac{h^{2} \sigma^{5}}{32} u_{i+1}^{n-1} u_{i+1}^{n}+\frac{3 h^{2} \sigma^{5}}{32}\left(u_{i+1}^{n}\right)^{2} .
\end{aligned}
$$

Next, we discretize the diffusion term,

$$
\int_{x_{i}}^{x_{i}+\sigma h} \int_{x_{i}-\sigma h}^{x_{i}} \int_{s_{1}}^{s_{2}} \varepsilon \frac{\partial^{2}\left(u^{n+\frac{1}{2}}\right)}{\partial x^{2}} d x d s_{1} d s_{2}=\varepsilon h \sigma\left(u_{i+\beta}^{n+\frac{1}{2}}-2 u_{i}^{n+\frac{1}{2}}+u_{i-\alpha}^{n+\frac{1}{2}}\right) .
$$

Similarly, we need to use the interpolation functions to approximate $u_{i+\beta}^{n+\frac{1}{2}}$ and $u_{i-\alpha}^{n+\frac{1}{2}}$. We obtain,

$$
\begin{aligned}
& u_{i+\beta}^{n+\frac{1}{2}}=\frac{\sigma(\sigma-1)}{2} u_{i-1}^{n+\frac{1}{2}}-\left(\sigma^{2}-1\right) u_{i}^{n+\frac{1}{2}}+\frac{\sigma(\sigma+1)}{2} u_{i+1}^{n+\frac{1}{2}} . \\
& u_{i-\alpha}^{n+\frac{1}{2}}=\frac{\sigma(\sigma+1)}{2} u_{i-1}^{n+\frac{1}{2}}+\left(\sigma^{2}-1\right) u_{i}^{n+\frac{1}{2}}+\frac{\sigma(\sigma-1)}{2} u_{i+1}^{n+\frac{1}{2}} .
\end{aligned}
$$

To substitute Eq.(14) and Eq.(15) into Eq.(13). Combining Eq.(7), we get

$$
\int_{x_{i}}^{x_{i}+\sigma h} \int_{x_{i}-\sigma h}^{x_{i}} \int_{s_{1}}^{s_{2}} \varepsilon \frac{\partial^{2}\left(u^{n+\frac{1}{2}}\right)}{\partial x^{2}} d x d s_{1} d s_{2}=B_{0} u_{i-1}^{n+1}+B_{1} u_{i}^{n+1}+B_{2} u_{i+1}^{n+1}+B_{3} .
$$

in which $B_{3}=B_{4} u_{i-1}^{n}+B_{5} u_{i}^{n}+B_{6} u_{i+1}^{n}, B_{0}=-\frac{1}{2} B_{1}=B_{2}=B_{4}=-\frac{1}{2} B_{5}=B_{6}=\frac{\sigma^{3} \varepsilon h}{2}$

Here we obtain the discrete form of the diffusion term.

Finally, we need to obtain the discrete form of the time term.

By Eq.(6) the term can be written,

$$
\int_{x_{i}}^{x_{i}+\sigma h} \int_{x_{i}-\sigma h}^{x_{i}} \int_{s_{1}}^{s_{2}}\left(\frac{\partial u}{\partial t}\right)^{n+\frac{1}{2}} d x d s_{1} d s_{2}=\int_{x_{i}}^{x_{i}+\sigma h} \int_{x_{i}-\sigma h}^{x_{i}} \int_{s_{1}}^{s_{2}} \frac{u^{n+1}-u^{n}}{\tau} d x d s_{1} d s_{2} .
$$

and the Lagrange expansions for $u^{n+1}$ and $u^{n}$ are shown as

$$
u^{q}=\frac{\left(x-x_{i}\right)\left(x-x_{i+1}\right)}{\left(x_{i-1}-x_{i}\right)\left(x_{i-1}-x_{i+1}\right)} u_{i-1}^{q}+\frac{\left(x-x_{i-1}\right)\left(x-x_{i+1}\right)}{\left(x_{i}-x_{i-1}\right)\left(x_{i}-x_{i+1}\right)} u_{i}^{q}+\frac{\left(x-x_{i-1}\right)\left(x-x_{i}\right)}{\left(x_{i+1}-x_{i-1}\right)\left(x_{i+1}-x_{i}\right)} u_{i+1}^{q}, \quad(q=n, n+1)
$$

To substitute Eq.(18) into Eq. (17), we can obtain the corresponding discrete form,

$$
\int_{x_{i}}^{x_{i}+\sigma h} \int_{x_{i}-\sigma h}^{x_{i}} \int_{s_{1}}^{s_{2}} \frac{u^{n+1}-u^{n}}{\tau} d x d s_{1} d s_{2}=C_{0} u_{i-1}^{n+1}+C_{1} u_{i}^{n+1}+C_{2} u_{i+1}^{n+1}+C_{3} \text {. }
$$

where $C_{3}=C_{4} u_{i-1}^{n}+C_{5} u_{i}^{n}+C_{6} u_{i+1}^{n}, C_{0}=-C_{4}=\frac{h^{3} \sigma^{5}}{12 \tau}, C_{1}=-C_{5}=-\frac{h^{3} \sigma^{3}}{6 \tau}\left(-6+\sigma^{2}\right), C_{2}=-C_{6}=\frac{h^{3} \sigma^{5}}{12 \tau}$

Combining (12), (16) and (19) for a one-dimensional transient nonlinear Burgers equation, a new discrete scheme is obtained.

$$
A u_{i-1}^{n+1}+B u_{i}^{n+1}+C u_{i+1}^{n+1}+D=0 .
$$

in which $A=A_{0}+C_{0}-B_{0}, B=A_{1}+C_{1}-B_{1}, C=A_{2}+C_{2}-B_{2}$

$$
D=A_{3}+\left(C_{4}-B_{4}\right) u_{i-1}^{n}+\left(C_{5}-B_{5}\right) u_{i}^{n}+\left(C_{6}-B_{6}\right) u_{i+1}^{n}
$$

According to Eq.(20) we can see that this method contains a temporal three layers' discrete scheme. The function values of the $n+1$ layer nodes can be obtained through the $n-1$ and $n$ layer. That is to say, in the case of determining initial conditions and boundary conditions, we need two layers to start the format. We use the prediction correction method to obtain the values of second layer according to the initial conditions, and then obtain the values of all nodes. The prediction correction method is shown as follows: 
$\begin{array}{cc}\text { Predictor : } & \overline{u^{n+1}}=u^{n}+\tau\left(-u^{n} \frac{\partial u^{n}}{\partial x}+\varepsilon \frac{\partial^{2} u^{n}}{\partial x^{2}}\right) . \\ \text { Corrector : } & u^{n+1}=u^{n}+\frac{\tau}{2}\left[\left(-u^{n} \frac{\partial u^{n}}{\partial x}+\varepsilon \frac{\partial^{2} u^{n}}{\partial x^{2}}\right)+\left(-\overline{u^{n+1}} \frac{\partial \overline{u^{n+1}}}{\partial x}+\varepsilon \frac{\partial^{2} \overline{u^{n+1}}}{\partial x^{2}}\right)\right] .\end{array}$

\section{Numerical example}

Problem: The solution of Eq.(1) on $0 \leq x \leq 1$ with initial condition $u(x, 0)=\sin (x \pi)$, with boundary conditions $u(0, t)=u(1, t)=0, t>0$ are considered. The exact solution is obtained by [2].

$$
u(x, t)=2 \pi \varepsilon \frac{\sum_{n=1}^{\infty} c_{n} \exp \left(-n^{2} \pi^{2} \varepsilon t\right) n \sin (n \pi x)}{c_{0}+\sum_{n=1}^{\infty} c_{n} \exp \left(-n^{2} \pi^{2} \varepsilon t\right) \cos (n \pi x)}
$$

where $c_{0}=\int_{0}^{1} \exp \left(-\frac{1-\cos (\pi x)}{2 \pi \varepsilon}\right) d x, c_{n}=2 \int_{0}^{1} \exp \left(-\frac{1-\cos (\pi x)}{2 \pi \varepsilon}\right) \cos (n \pi x) d x \quad(n=1,2,3, \mathrm{~L}), \varepsilon=1$.

From Table.1 we can see clearly that the finite volume method in this paper is closer to the exact solution than the other three methods when taking the same time and space step size. Moreover, by adjusting the value of $\sigma$, we can obtain better numerical results.

Table 1 Comparison of results at different times and nodes for $\mathrm{v}=1, d t=0.0001, h=0.0125$.

\begin{tabular}{cllllll}
\hline$x$ & $t$ & HC[5] & RHC[5] & RPA[2] & Current & Exact \\
\hline 0.25 & 0.10 & 0.252942 & 0.264126 & 0.252875 & 0.253644 & 0.253637 \\
& 0.15 & 0.156059 & 0.165683 & 0.155447 & 0.156607 & 0.156601 \\
& 0.20 & 0.095889 & 0.101617 & 0.094289 & 0.096447 & 0.096440 \\
& 0.25 & 0.056174 & 0.059113 & 0.055674 & 0.059222 & 0.059217 \\
\hline 0.50 & 0.10 & 0.376474 & 0.393354 & 0.373085 & 0.371588 & 0.371579 \\
& 0.15 & 0.235875 & 0.251788 & 0.228940 & 0.226833 & 0.226825 \\
& 0.20 & 0.153645 & 0.163931 & 0.142127 & 0.138481 & 0.138473 \\
& 0.25 & 0.112810 & 0.120967 & 0.091944 & 0.084543 & 0.084537 \\
\hline 0.75 & 0.10 & 0.271517 & 0.285579 & 0.272368 & 0.272589 & 0.272580 \\
& 0.15 & 0.162739 & 0.176957 & 0.163628 & 0.164376 & 0.164369 \\
& 0.20 & 0.098431 & 0.111020 & 0.098656 & 0.099441 & 0.099434 \\
& 0.25 & 0.057394 & 0.068569 & 0.059343 & 0.060351 & 0.060346 \\
\hline
\end{tabular}

\section{Conclusions}

In this paper, a new discrete scheme is constructed for nonlinear transient Burgers equation by using the multiple integral finite volume method combined with Lagrange interpolation method. The effectiveness and superiority of the proposed scheme are demonstrated by comparing numerical results with other schemes. The value of $\sigma$ can be adjusted basing on the needs of project to meet the engineering requirements. In space, we use three-points Lagrange interpolation method to achieve the third order accuracy, and we can increase interpolation nodes to get higher accuracy. All in all, the numerical method in this paper has a good application prospect.

\section{References}

[1] J.M. Burger: Adv. in Appl. Mech. Vol. I (1948), p. 171-199.

[2] M. Gulsu: Appl. Math. Comput. Vol.175 (2006), p. 1245-1255.

[3] X.J. Yang and Y.B.Ge: Mathematics in Practice and Theory, Vol. 11(2016), p.036.

[4] J. Li, F. Liu, L. Feng and I. Turner: Comput. Math. Appl. Vol. 05 (2017), p.017.

[5] M. Gulsu and T. O” zis: Appl. Math. Comput. Vol. 01 (2005), p.106. 